\title{
What does matrix metalloproteinase-1 expression in patients with breast cancer really tell us?
}

\author{
Ferdinando Mannello
}

\begin{abstract}
Molecular and biochemical expressions of matrix metalloproteinases in breast cancer tissue and cells offers promise in helping us understand the breast cancer microenvironment, and also in the future it is hoped this will improve its detection, treatment and prognosis. In a retrospective study recently published in BMC Cancer, microenvironment predisposing to breast cancer progression, metastatic behavior and the expression of matrix metalloproteinase-1 (MMP-1) and its correlation with well-known biochemical, molecular and clinicopathologic factors in breast cancer cells and cancer-associated stromal cells was examined; this study also analyzed patient survival in different breast cancer subtypes. The positive correlation in breast tumor and stromal cells between MMP-1 expression and several markers of tumor grade and stage provide us with some useful new insights into important questions about the molecular profiling of the stromal microenvironment in metastatic breast cancer. The study showed that MMP-1 expression is strongly associated with poor clinical outcome, so now we look forward to future larger studies in breast cancer patients in which we can relate wider MMP molecular profiling to identify lethal tumor and stromal microenvironments predisposing to breast cancer progression, metastatic behavior and poor prognosis.

Please see related article http://www.biomedcentral. $\mathrm{com} / 1471-2407 / 11 / 348$
\end{abstract}

\section{Introduction}

Breast cancer $(\mathrm{BC})$ is the most common cancer in women worldwide, comprising at least $16 \%$ of all female cancers. BC results from multiple environmental and hereditary risk factors, even though genetic traits, age

Correspondence: ferdinando.mannello@uniurb.it

Department of Biomolecular Sciences, Section of Clinical Biochemistry, Unit of Cell Biology, University 'Carlo Bo' of Urbino, I-61029 Urbino (PU), Italy and hormones are the main recognized $\mathrm{BC}$-predisposing risk factors [1]. Human female $\mathrm{BC}$ encompasses a variety of tumors, which differ in their morphological, biochemical and molecular characteristics, all guiding clinical outcome and patient survival. Although welldocumented classic diagnostic/prognostic biomarkers/ profiles are reliable (for example, tumor grade and stage, p53, bcl-2, Ki-67, hormone receptor status,: human epidermal growth factor receptor 2 (HER-2) expression), there is the urgent need to differentiate between $B C$ subclasses (for example, non-basal-like luminal A and B, basal-like, triple-negative $\mathrm{BC})[2,3]$, patients with different prognoses and treatment responses to the same therapy [4-6].

Examining new BC biomarkers has proven that matrix metalloproteinases (MMP), which are zincdependent endopeptidases belonging to the Metzincin superfamily, are involved in several key events of both physiologic processes (for example, tissue remodelling, stem cell differentiation and proliferation, apoptosis) [7-11] and in pathological conditions (for example, inflammation, degeneration and cancer) [12-14]. The MMP family comprises several classes of proteases [15], which cleave almost all extracellular matrix components and a variety of proteins and growth factors crucial for neoplastic initiation and progression; these data suggest MMPs as good targets for tumor biomarker discovery. In humans, there are 24 MMP genes, but only 23 MMP proteins [16], including 17 soluble, secreted enzymes and 6 membrane-associated proteinases. MMPs are built up by a diverse structural domain architecture, and differ in their substrate specificity and in temporal and tissue specific expression patterns. MMPs were originally named for their preferred substrates within the extracellular matrix (ECM): collagen-cleaving MMPs (MMP-1, -8, and -13) were designated collagenases, gelatin (denatured collagen)-cleaving MMPs (MMP-2 and -9) were termed gelatinases, and MMPs degrading a broad spectrum of ECM proteins were called stromelysins (MMP-3, -10, 
and -11) or matrilysins (MMP-7). As the MMP family grew with the discovery of additional paralogs, including the membrane-associated MMPs, a numbering system was adopted, and MMPs are now grouped according to their domain structure (Figure 1).

Many studies have characterized the increased expression of MMPs at both protein and mRNA levels, identifying them as a key event leading to the initiation/ progression of $\mathrm{BC}$ and linking them with the ability of cancer to metastasize. Thus, MMPs could be used as tumor biomarkers and indicators of cancer metastasis with diagnostic and prognostic usefulness $[17,18]$. On the other hand, recent evidence underlines the fact that some MMPs (such as MMP-8, also named collagenase-2 or neutrophil collagenase) may favor host defense instead of stimulating tumor proliferation, suggesting that these proteinases have an unexpected protective biological role in cancer processes $[19,20]$.

Among the MMP members involved in $\mathrm{BC}$, both the biochemical and molecular expression profile of MMP-1 (named also collagenase-1 or interstitial collagenase) have been extensively analyzed in human BC. Although there are a great number of valuable in vitro and in vivo studies concerning its role in breast carcinogenesis (see reviews [21-23]), some parts of its regulation and expression (as an assisting marker in metastatic BC diagnosis) remain poorly understood and a matter of debate $[21,24,25]$.

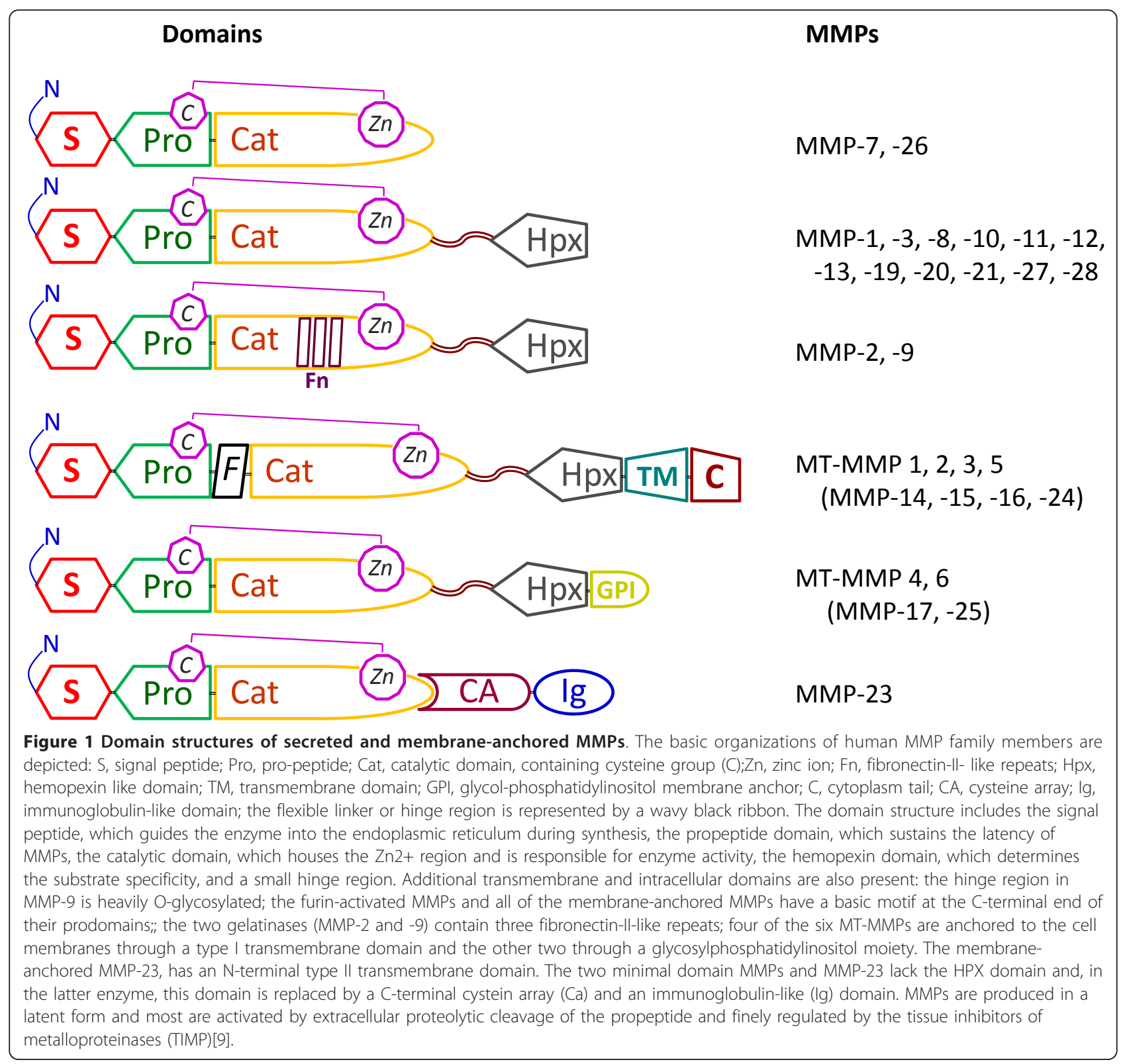


To address this hot topic, a recent study published in $B M C$ Cancer [26] has retrospectively examined MMP-1 expression in both breast cancer cells and cancer-associated stromal cells from $\mathrm{BC}$ patient to evaluate the relationship among MMP-1 and classic prognostic factors, analyzing the extensively long follow-up time for cancer specific survival in different BC subtypes.

\section{Discussion}

The highly complex BC tissue is composed of neoplastic cells and stromal cell compartments [27], containing a variety of mesenchymal cells (notably fibroblasts, myofibroblasts, endothelial cells and inflammatory cells associated with the immune system) (Figure 2). The specific contributions of the cancer-associated fibroblasts to tumor growth are poorly understood, but it has been suggested that they are able to promote the growth of mammary carcinoma cells and to enhance tumor angiogenesis [28-30], as well as through the secretion of proteinases (including MMP-1) and other proteins (for example, stromal-cell derived factor 1 (also called CXCL 12), syndecan-1, CXCR4 and Caveolin-1) [31,32]. The molecular profile of the lethal breast cancer microenvironment is based on activated cancer-associated

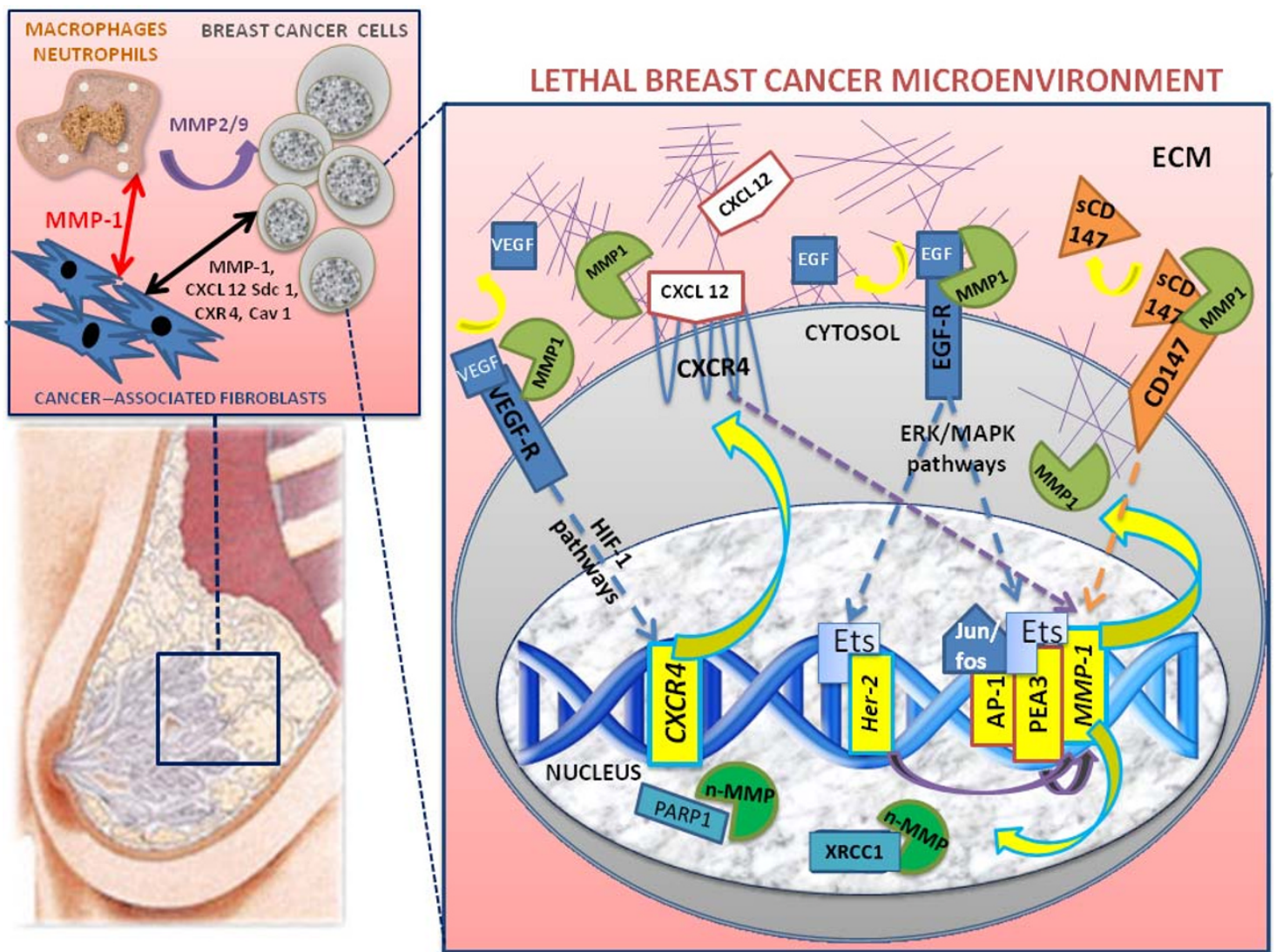

Figure 2 Schematic view of the role and functions of MMP-1 in breast cancer microenvironment. The breast microenvironment is composed of ductal and luminal epithelial cells sitting on a layer of myoepithelial cells, and stromal cells (including leukocytes, fibroblasts, and endothelial cells). The microenvironment alterations during breast cancer (BC) progression lead to a decrease of myoepithelial cells, phenotypically altered because of signals coming from the tumor and stromal cells. The cell-cell and cell-matrix interactions during BC initiation/ progression involve the interplay among cancer-associated fibroblasts, macrophages and BC cells. The secretion of MMP-1 and the release of soluble proteins (including EGF, VEGF, CD147, syndecan-1) in the breast milieuare able to activate tumor pathways, generating the 'lethal BC microenvironment', which triggers a 'vicious cycle' between tumor and stromal cells, enhancing the BC growth, and promoting the invasive and metastatic processes via paracrine interactions [13]. The unexpected roles of MMP-1 interstitial collagenase are related to the proteolytic activity in the ECM compartment and the cell-cell interactions. The unexpected nuclear localization of some MMPs has been recently linked to new functional roles of these proteases within nuclei of tumor cells, with the ability to cleave peculiar nuclear peptides (Mannello et al, ms in preparation). The proteolytic activity of MMP-1 in BC cells is linked to the cleavage/release of soluble VEGF, EGF, CXCL 12 and CD147 from their receptor complexes. These proteins are able to activate crucial cancer metabolic routes (like HIF-1-dependent and MAPK and ERK-dependent pathways) that promote BC proliferation, triggering a vicious cycle through the molecular activation of some transcription factors (like AP-1, PEA3 and Ets), indispensable switches for the HER-2, CXCR4 and MMP-1 gene regulation [39]. The secretion of MMP-1 is also able to activate other MMPs (like MMP-2 and -9) which have been widely recognized as crucial steps for the BC evolution [14]. 
fibroblasts and revealed also by high levels of interstitial collagenase MMP-1 (both at protein and mRNA levels), which facilitate angiogenesis and increase ECM degradation, both crucial processes for the invasive and migratory phenotype of metastatic BC [14]. Interestingly, it has also been demonstrated that human epidermal growth factor receptor 2 (HER-2) induces MMP-1 expression through the enhanced regulation of the transcription factor/proto-oncoprotein Ets-1, suggesting that (contrary to the protective role of MMP- 8 neutrophil collagenase [20]) the interstitial collagenase MMP-1 may significantly affect the metastatic behavior of $\mathrm{BC}$ cells [33]. The same biochemical behaviors and biological functions of both MMP-1 and - 8 collagenases have also been detected in plasma samples collected from BC patients with poor prognosis [34].

The recent study of Boström and Colleagues [26] is helpful in that it sought to link the expression of MMP1 with a broader picture of immunohistochemical biomarker changes, and their relationships between $\mathrm{BC}$ and stromal cells. According to the well-established role of MMP-1 in BC promotion and outcome [21,35], the authors claim that the independent prognostic value of MMP-1 is mainly based on the positive correlations with tumor grade and p53 positivity in tumor and stromal cells; the peculiar expression of MMP-1 in stromal cells also showed a significant association of BC with HER-2 over-expression and triple negative BC. Furthermore, in the luminal B BC subtype (estrogen receptor and/or progesterone receptor positive, other than HER2 positive), MMP-1 expression in stromal cells was higher than in the luminal A subtype (estrogen receptor and/or progesterone receptor positive, and HER-2 negative). Also, the luminal B subtype stromal cells showed higher MMP-1 expression when compared to triple negative $\mathrm{BC}$ cells (identified as a basal-like subtype in about $70 \%$ of cases). From the more than 20 years survival analyses, Boström and colleagues revealed that there were statistically significant differences in BC-specific survival among women with tumors with high versus low expression of MMP-1, tumor grade I versus III, triple-negative versus non-triple negative, basal-like versus non-basal-like tumors, and low versus high Bcl-2 and Ki-67 expressions. All these data reveal that high MMP1 positivity in both stromal and tumor cells was significantly associated with tumor evolution, poor prognosis and shortened survival. Noteworthy also is the higher MMP-1 expression in both the cytoplasm and nuclei of $\mathrm{BC}$ cells with respect to stromal cells, adding new information to the poorly characterized and unexpected role of nuclear MMPs (Mannello et al, unpublished observations), suggesting them not just for ECM anymore [11]. In this respect, it has recently been suggested that some MMPs (such as the MMP-3 stromelysin and MMP-2 gelatinase) may be involved in transcriptional gene regulation [36] and the cleavage of poly-ADP-ribose-polymerase [37].

Recent studies highlight the biological importance of stroma in breast physiopathology [38], suggesting that the gene expression and regulation of some MMPs are largely restricted to the stromal compartment [39]. Although the role of the stromal compartment in BC had been originally depicted as the Cinderella of the cancer biology, even more evidence has supported the crucial role of stromal cells in tumor evolution and then in $\mathrm{BC}$ patient survival. Early studies showed that the normal mammary microenvironment is capable of reverting the malignant phenotype of $\mathrm{BC}$ cells by inducing a more differentiated state, suggesting that cancer cells may only thrive in an abnormal environment in which they evolved [40]. Pathologists have also long noted the prognostic value of certain histopathological features of BC (including lymphocytic infiltration and angiogenesis), suggesting a role for non-epithelial cells in carcinogenesis, as shown by differences in tumor initiation and progression depending on the variability in germline genotypes and phenotypes [41].

Despite the convincing observations implicating a role for microenvironmental and systemic alterations in breast tumorigenesis (reviewed in [42]), our understanding of the genes and metabolic pathways mediating cellular interactions and paracrine regulatory networks among various cell types in both normal and neoplastic breast tissue is still limited. However, it has been clearly demonstrated that gene expression changes occur in all cell types during breast tumor progression, but clonally selected genetic alterations are restricted to tumor epithelial cells [43]. Interestingly, the comparison of myoepithelial cells from normal breast tissue with ductal carcinoma in situ (DCIS) yielded the highest number of consistently differentially expressed genes; a significant fraction of these encoded for secreted proteins and cell surface receptors, suggesting intensive autocrine/paracrine regulatory loops in the breast pre-cancer and cancer microenvironment $[27,30,31,42]$.

It has recently been demonstrated that the gene expression signature of epithelial cells correlated with tumor grade but not with histologic stage, whereas genes up-regulated in tumor-associated stroma included many ECM-related molecules (including MMP-1 collagenase), expressed at higher levels in invasive compared with in situ tumors [44,45]. In particular, it has been recognized that, among the mesenchymal cells constituting the stroma, both the cancer-associated fibroblasts and the inflammatory cells associated with the immune system are able to drive cancer initiation and progression through peculiar biochemical pathways and molecular signatures, significantly different between 
in situ and invasive BC $[42,46]$. The dramatic changes in gene expression patterns, but the lack of clonally selected somatic genetic alterations in the tumor-associated myoepithelial and stromal cells, has also suggested potential epigenetic alterations; this may be because stromal cells isolated from normal and tumor tissues are known to maintain their differences even after prolonged cell culture and in xenograft studies (as reviewed in [38]).

The historically prevailing view of $\mathrm{BC}$ progression is focused on tumor epithelial cells, whereby gradual progression of a tumor through defined steps is entirely due to the accumulation of both genetic and epigenetic alterations that confer progressively malignant phenotypes [47]. However, this model has been challenged after multiple studies demonstrating the importance of the microenvironment in shaping tumor evolution and progression through the new functions of several MMPs (including MMP-1 collagenase)[42,47-50].

With this background, the molecular and biochemical profiling of a lethal $\mathrm{BC}$ microenvironment has led to the identification of new functions of MMP-1 in the stromal microenvironment of $\mathrm{BC}$, revealing a significant link between cancer-associated fibroblasts and MMP-1 expression with metastatic tumor progression and/or poor clinical outcome [21]. In particular, the paper of Boström and colleagues [26] sheds further light on these relationships, demonstrating significant differences of MMP-1 expression by cancer-associated stromal cells in luminal A, luminal B and triple-negative $\mathrm{BC}$ subclasses. The importance of MMP-1 expression and its cellular localization (other than the unexpected presence of nuclear MMP-1) in BC stromal cells is in agreement with the functional and clinical relevance of microenvironmental alterations in breast tumorigenesis. Their results support the molecular evidence that high MMP1 mRNA expression and both aplotypes and polymorphisms of MMP-1 promoter gene may represent a risk factor in patients with invasive $\mathrm{BC}$ [51], and recognize MMP-1 as a prognostic marker in patients with invasive/metastatic BC [52-54]. Thus, Boström's study provides stronger evidence that the expression of MMP-1 in stromal fibroblasts of $\mathrm{BC}$ help to identify patients with atypical tumor-stromal fibroblasts in which MMP1 may contribute to $B C$ invasiveness and metastatic behavior.

\section{Conclusions}

Boström and colleagues provide us with a stronger scientific basis for understanding the involvement and cooperation of both tumor and stromal cells in BC progression and outcome, as well as the role of MMP-1 expression (both in cytoplasm and nuclei) for metastatic dissemination, identifying $\mathrm{BC}$ women with shortened relapse-free survival and poor outcome.

Although we are now learning that the useful relationship among MMP-1 expression and well-known clinic/ pathologic-prognostic factors (such as Ki-67, HER-2, Bcl-2, tumor grade and cancer subtypes) may help us to enhance and increase the clinically useful prognostic factors, what we need are future biochemical, molecular and clinical studies assessing many different biomarkers head-to-head, because MMP-1 analysis alone is no longer the way to go.

In this respect, the perspectives should involve studies focused on gene and protein expression profiling of stromal alterations associated with $\mathrm{BC}$ progression, identifying key transcriptional changes that occur early in cancer initiation/development. For example, a possible synopsis for future research could include:

1. The profile of the MMP superfamily (including the proteomic analysis of both protective and dangerous MMPs). This research will evaluate the kind of MMP which is involved in the proteolytic cascade cleaving/ regulating not only structural components of both ECM and nuclear compartments, but also the modulation of several growth factor precursors, cell surface receptors, cytokines and cell adhesion molecules; on the other hand, we should obtain information about the MMPs involved in tumor-suppression [20].

2. The analysis of tissue inhibitors of metalloproteinase (TIMPs) (well-known inhibitors of MMPs also characterized by MMP-independent functions in cancer biology $[9,55])$. This approach allows the analysis of the proteolytic/antiproteolytic balance, as a cancer/metastatic specific switch). It will be interesting for future studies to identify processes that control the earlier stages of disease progression, helping to decipher the molecular and biochemical mechanisms underlying cellular and microenvironmental interactions in the breast tumors.

3. The screening of both MMP gene aplotypes and polymorphisms, to obtain a phamacogenomic profile of the individual variation in drug response and therapeutic efficacy [56]. Considering the potential contribution of BC stromal microenvironmental alterations on MMP-1 biochemical and molecular alterations, the knowledge of MMP gene aplotypes and/or polymorphisms may help in the design of more efficient target therapy, limiting drug-resistance and underlining the importance of antitarget identification in drug development for blocking metastases [14].

4. The histochemical and molecular analysis of the nuclear localization of MMP-1 protein (in latent and/or active form) to evaluate its role during cancer associated apoptosis and possible therapeutic potential (Mannello 
et al, unpublished observations and manuscript in preparation).

As highlighted by Boström and colleagues, MMP-1 expression in both stromal and tumor cells may control $\mathrm{BC}$ progression, suggesting that $\mathrm{BC}$ metastasis and outcome are driven by complex and reciprocal interactions between epithelial cancer cells and their stromal microenvironment. The biochemical and molecular profiling of invasive $\mathrm{BC}$ will be crucial to identify a 'lethal tumor microenvironment' [57] associated with metastatic tumor progression and/or poor clinical outcome for the leading cause of cancer death among women in highincome countries [1]. It is noteworthy that the 'lethal tumor microenvironment' is likely to have an impact on numerous solid tumors in different parts of the body, widening the impact of the results of Boström and colleagues to other fields of oncology (such as in breast, lung, pancreas and haematological malignancies)[50,58]. In fact, the tumor microenvironment alterations in human cancer not only influence tumor progression and predict prognosis, but also have major effects on the efficacy of cancer therapy, especially the targeted therapy aimed at growth factor receptors and secreted proteins, such as HER-2 and MMP-1. Modifications in the cancer microenvironment may alter the fitness landscape providing a possible growth advantage for cells with tumorinitiating genetic-epigenetic changes [59,60].

Ultimately, the study of Boström and Colleagues opens new possibilities and the ability to specifically target the expression of MMP-1, a particular MMP involved in $\mathrm{BC}$ initiation/progression, aberrantly expressed in the metastatic process, suggesting further diagnostic, prognostic and therapeutic potential.

\section{Abbreviations}

BC: breast cancer; DCIS: ductal carcinoma in situ; HER-2: human epidermal growth factor receptor 2; MMP: matrix metalloproteinase; TIMP: tissue inhibitors of metalloproteinase.

\section{Competing interests}

The author declares that they have no competing interests.

Received: 9 June 2011 Accepted: 11 August 2011

Published: 11 August 2011

\section{References}

1. Jemal A, Bray F, Center MM, Ferlay J, Ward E, Forman D: Global cancer statistics. CA Cancer J Clin 2011, 61:69-90.

2. Sorlie T: Molecular portraits of breast cancer: tumour subtypes as distinct disease entities. Eur J Cancer 2004, 40:2667-2675.

3. Sorlie T: Introducing molecular subtyping of breast cancer into the clinic? J Clin Oncol 2009, 27:1153-1154.

4. Sorlie T: Molecular classification of breast tumors: toward improved diagnostics and treatments. Methods Mol Biol 2007, 360:91-114.

5. Borgan E, Sitter B, Lingjaerde OC, Johnsen H, Lundgren S, Bathen TF, Sorlie T, Borresen-Dale AL, Gribbestad IS: Merging transcriptomics and metabolomics-advances in breast cancer profiling. BMC Cancer 2010, 10:628.
6. Zhao X, Rodland EA, Sorlie T, Naume B, Langerod A, Frigessi A, Kristensen VN, Borresen-Dale AL, Lingjaerde OC: Combining gene signatures improves prediction of breast cancer survival. PLoS One 2011, 6:e17845.

7. Mannello F: Natural bio-drugs as matrix metalloproteinase inhibitors: new perspectives on the horizon? Recent Pat Anticancer Drug Discov 2006, 1:91-103.

8. Mannello F, Luchetti F, Falcieri E, Papa S: Multiple roles of matrix metalloproteinases during apoptosis. Apoptosis 2005, 10:19-24.

9. Mannello F, Gazzanelli G: Tissue inhibitors of metalloproteinases and programmed cell death: conundrums, controversies and potential implications. Apoptosis 2001, 6:479-482.

10. Tonti GA, Mannello F, Cacci E, Biagioni S: Neural stem cells at the crossroads: MMPs may tell the way. Int J Dev Biol 2009, 53:1-17.

11. Hadler-Olsen E, Fadnes B, Sylte I, Uhlin-Hansen L, Winberg JO: Regulation of matrix metalloproteinase activity in health and disease. FEBS J 2011, 278:28-45

12. Mannello F, Tonti G, Papa S: Matrix metalloproteinase inhibitors as anticancer therapeutics. Curr Cancer Drug Targets 2005, 5:285-298.

13. Kessenbrock K, Plaks V, Werb Z: Matrix metalloproteinases: regulators of the tumor microenvironment. Cell 2010, 141:52-67.

14. Gialeli C, Theocharis AD, Karamanos NK: Roles of matrix metalloproteinases in cancer progression and their pharmacological targeting. FEBS J 2011, 278:16-27.

15. Cauwe B, Opdenakker G: Intracellular substrate cleavage: a novel dimension in the biochemistry, biology and pathology of matrix metalloproteinases. Crit Rev Biochem Mol Biol 2010, 45:351-423.

16. Yan C, Boyd DD: Regulation of matrix metalloproteinase gene expression. J Cell Physiol 2007, 211:19-26.

17. Roy R, Yang J, Moses MA: Matrix metalloproteinases as novel biomarkers and potential therapeutic targets in human cancer. J Clin Oncol 2009, 27:5287-5297.

18. Delassus GS, Cho H, Hoang S, Eliceiri GL: Many new down- and upregulatory signaling pathways, from known cancer progression suppressors to matrix metalloproteinases, differ widely in cells of various cancers. J Cell Physiol 2010, 224:549-558.

19. Gutierrez-Fernandez A, Fueyo A, Folgueras AR, Garabaya C, Pennington CJ, Pilgrim S, Edwards DR, Holliday DL, Jones JL, Span PN, Sweep FC,

Puente XS, López-Otín C: Matrix metalloproteinase-8 functions as a metastasis suppressor through modulation of tumor cell adhesion and invasion. Cancer Res 2008, 68:2755-2763.

20. Lopez-Otin C, Palavalli LH, Samuels Y: Protective roles of matrix metalloproteinases: from mouse models to human cancer. Cell Cycle 2009, 8:3657-3662.

21. MCGowan PM, Duffy MJ: Matrix metalloproteinase expression and outcome in patients with breast cancer: analysis of a published database. Ann Oncol 2008, 19:1566-1572.

22. Eltarhouny SA, Elsawy WH, Radpour R, Hahn S, Holzgreve W, Zhong XY: Genes controlling spread of breast cancer to lung "gang of 4". Exp Oncol 2008, 30:91-95.

23. Eck SM, Blackburn JS, Schmucker AC, Burrage PS, Brinckerhoff CE: Matrix metalloproteinase and $\mathrm{G}$ protein coupled receptors: co-conspirators in the pathogenesis of autoimmune disease and cancer. J Autoimmun 2009, 33:214-221.

24. Baker AH, Edwards DR, Murphy G: Metalloproteinase inhibitors: biological actions and therapeutic opportunities. J Cell Sci 2002, 115:3719-3727.

25. Kohrmann A, Kammerer U, Kapp M, Dietl J, Anacker J: Expression of matrix metalloproteinases (MMPs) in primary human breast cancer and breast cancer cell lines: New findings and review of the literature. BMC Cancer 2009, 9:188.

26. Boström $P$, Soderstrom M, Vahlberg $T$, Soderstrom KO, Roberts PJ, Carpen O, Hirsimaki P: MMP-1 expression has an independent prognostic value in breast cancer. BMC Cancer 2011.

27. Orimo A, Weinberg RA: Heterogeneity of stromal fibroblasts in tumors. Cancer Biol Ther 2007, 6:618-619.

28. Hasebe $Y$, Egawa K, Shibanuma M, Nose K: Induction of matrix metalloproteinase gene expression in an endothelial cell line by direct interaction with malignant cells. Cancer Sci 2007, 98:58-67.

29. Gao MQ, Kim BG, Kang S, Choi YP, Park H, Kang KS, Cho NH: Stromal fibroblasts from the interface zone of human breast carcinomas induce 
an epithelial-mesenchymal transition-like state in breast cancer cells in vitro. J Cell Sci 2010, 123:3507-3514.

30. Hasebe T, Iwasaki M, kashi-Tanaka S, Hojo T, Shibata T, Sasajima Y, Kinoshita T, Tsuda H: Atypical tumor-stromal fibroblasts in invasive ductal carcinoma of the breast. Am J Surg Pathol 2011, 35:325-336.

31. Orimo A, Weinberg RA: Stromal fibroblasts in cancer: a novel tumorpromoting cell type. Cell Cycle 2006, 5:1597-1601.

32. ECk SM, Cote AL, Winkelman WD, Brinckerhoff CE: CXCR4 and matrix metalloproteinase-1 are elevated in breast carcinoma-associated fibroblasts and in normal mammary fibroblasts exposed to factors secreted by breast cancer cells. Mol Cancer Res 2009, 7:1033-1044.

33. Park YH, Jung HH, Ahn JS, Im YH: Ets-1 upregulates HER2-induced MMP-1 expression in breast cancer cells. Biochem Biophys Res Commun 2008, 377:389-394.

34. Decock J, Hendrickx W, Vanleeuw U, V Van B, Van HS, Christiaens MR, Ye S, Paridaens R: Plasma MMP1 and MMP8 expression in breast cancer: protective role of MMP8 against lymph node metastasis. BMC Cancer 2008, 8:77.

35. Eck SM, Hoopes PJ, Petrella BL, Coon Cl, Brinckerhoff CE: Matrix metalloproteinase-1 promotes breast cancer angiogenesis and osteolysis in a novel in vivo model. Breast Cancer Res Treat 2009, 116:79-90.

36. Eguchi T, Kubota S, Kawata K, Mukudai Y, Uehara J, Ohgawara T, Ibaragi S, Sasaki A, Kuboki T, Takigawa M: Novel transcription-factor-like function of human matrix metalloproteinase 3 regulating the CTGF/CCN2 gene. Mol Cell Biol 2008, 28:2391-2413.

37. Aldonyte R, Brantly M, Block E, Patel J, Zhang J: Nuclear localization of active matrix metalloproteinase-2 in cigarette smoke-exposed apoptotic endothelial cells. Exp Lung Res 2009, 35:59-75.

38. Polyak K, Kalluri $\mathrm{R}$ : The role of the microenvironment in mammary gland development and cancer. Cold Spring Harb Perspect Biol 2010, 2:a003244.

39. Yan C, Boyd DD: Regulation of matrix metalloproteinase gene expression. J Cell Physiol 2007, 211:19-26.

40. DeCosse JJ, Gossens CL, Kuzma JF, Unsworth BR: Breast cancer: induction of differentiation by embryonic tissue. Science 1973, 181:1057-1058.

41. Wallace JA, Li F, Leone G, Ostrowski MC: Pten in the breast tumor microenvironment: modeling tumor-stroma coevolution. Cancer Res 2011, 71:1203-1207.

42. Cichon MA, Degnim AC, Visscher DW, Radisky DC: Microenvironmental influences that drive progression from benign breast disease to invasive breast cancer. J Mammary Gland Biol Neoplasia 2010, 15:389-397.

43. Allinen M, Beroukhim R, Cai L, Brennan C, Lahti-Domenici J, Huang H, Porter D, Hu M, Chin L, Richardson A, Schnitt S, Sellers WR, Polyak K: Molecular characterization of the tumor microenvironment in breast cancer. Cancer Cell 2004, 6:17-32.

44. Ma XJ, Dahiya S, Richardson E, Erlander M, Sgroi DC: Gene expression profiling of the tumor microenvironment during breast cancer progression. Breast Cancer Res 2009, 11:R7.

45. Brinckerhoff $C E$, Rutter $J$, Benbow U: Interstitial collagenases as markers of tumor progression. Clin Cancer Res 2000, 6:4823-4830.

46. Polyak K: Molecular markers for the diagnosis and management of ductal carcinoma in situ. J Natl Cancer Inst Monogr 2010, 2010:210-213.

47. Bissell MJ, Radisky D: Putting tumours in context. Nat Rev Cancer 2001, 1:46-54.

48. Stallings-Mann M, Radisky D: Matrix metalloproteinase-induced malignancy in mammary epithelial cells. Cells Tissues Organs 2007, 185:104-110.

49. Radisky DC, Bissell MJ: Matrix metalloproteinase-induced genomic instability. Curr Opin Genet Dev 2006, 16:45-50.

50. Bissell MJ, Kenny PA, Radisky DC: Microenvironmental regulators of tissue structure and function also regulate tumor induction and progression: the role of extracellular matrix and its degrading enzymes. Cold Spring Harb Symp Quant Biol 2005, 70:343-356.

51. Cheng S, Tada M, Hida Y, Asano T, Kuramae T, Takemoto N, Hamada J, Miyamoto M, Hirano S, Kondo S, Moriuchi T: High MMP-1 mRNA expression is a risk factor for disease-free and overall survivals in patients with invasive breast carcinoma. J Surg Res 2008, 146:104-109.

52. Pearce $E G$, Laxton RC, Pereira AC, Ye S: Haplotype effects on matrix metalloproteinase- 1 gene promoter activity in cancer cells. Mol Cancer Res 2007, 5:221-227.

53. Przybylowska K, Kluczna A, Zadrozny M, Krawczyk T, Kulig A, Rykala J, Kolacinska A, Morawiec Z, Drzewoski J, Blasiak J: Polymorphisms of the promoter regions of matrix metalloproteinases genes MMP-1 and MMP9 in breast cancer. Breast Cancer Res Treat 2006, 95:65-72.

54. Przybylowska K, Zielinska J, Zadrozny M, Krawczyk T, Kulig A, Wozniak P, Rykala J, Kolacinska A, Morawiec Z, Drzewoski J, Blasiak J: An association between the matrix metalloproteinase 1 promoter gene polymorphism and lymphnode metastasis in breast cancer. J Exp Clin Cancer Res 2004, 23:121-125.

55. Mellick AS, Blackmore D, Weinstein SR, Griffiths LR: An assessment of MMP and TIMP gene expression in cell lines and stroma - tumour differences in microdissected breast cancer biopsies. Tumour Biol 2003, 24:258-270.

56. Offit K, Robson ME: New pharmacogenomic paradigm in breast cancer treatment. J Clin Oncol 2010, 28:4665-4666.

57. Chen ST, Pan TL, Juan HF, Chen TY, Lin YS, Huang CM: Breast tumor microenvironment: proteomics highlights the treatments targeting secretome. J Proteome Res 2008, 7:1379-1387.

58. Kenny PA, Bissell MJ: Tumor reversion: correction of malignant behavior by microenvironmental cues. Int J Cancer 2003, 107:688-695.

59. Kenny PA, Lee GY, Bissell MJ: Targeting the tumor microenvironment. Front Biosci 2007, 12:3468-3474.

60. Kenny PA, Nelson CM, Bissell MJ: The Ecology of Tumors: By perturbing the microenvironment, wounds and infection may be key to tumor development. Scientist 2006, 20:30.

Pre-publication history

The pre-publication history for this paper can be accessed here: http://www.biomedcentral.com/1741-7015/9/95/prepub

doi:10.1186/1741-7015-9-95

Cite this article as: Mannello: What does matrix metalloproteinase-1 expression in patients with breast cancer really tell us? BMC Medicine 2011 9:95.

\section{Submit your next manuscript to BioMed Central and take full advantage of:}

- Convenient online submission

- Thorough peer review

- No space constraints or color figure charges

- Immediate publication on acceptance

- Inclusion in PubMed, CAS, Scopus and Google Scholar

- Research which is freely available for redistribution

Submit your manuscript at www.biomedcentral.com/submit
Biomed Central 